\title{
SRC Family Oncogene
}

National Cancer Institute

\section{Source}

National Cancer Institute. SRC Family Oncogene. NCI Thesaurus. Code C18540.

Human SRC Family Oncogenes are mutated variants of Tyrosine Kinase Family Genes, which encode diverse SRC Family Tyrosine Kinases, closely related intracellular nonreceptor protein-tyrosine kinases with amino acid sequence similarity to SRC and to Rous sarcoma virus oncogene protein PP60(V-SRC). SRC tyrosine kinases interact with diverse cell-surface receptors and participate in intracellular signal transduction pathways in a variety of hematopoietic cells. Oncogenic SRC kinases can occur through altered regulation or expression of the endogenous protein and by virally encoded src (v-src) genes. SRC family oncogenes disrupt normal cell function. 